\title{
Soil-Structure Interaction in Transversely Isotropic Layered Media Subjected to Incident Plane SH Waves
}

\author{
Zhenning $\mathrm{Ba}^{1,2}$ and Xi Gao ${ }^{3}$ \\ ${ }^{1}$ Department of Civil Engineering, Tianjin University, Tianjin 300072, China \\ ${ }^{2}$ Key Laboratory of Coast Civil Structure Safety, Tianjin University, Tianjin 300072, China \\ ${ }^{3}$ Tianjin International Engineering Institute, Tianjin University, Tianjin 300072, China \\ Correspondence should be addressed to Zhenning Ba; bazhenning_001@163.com
}

Received 3 March 2017; Revised 15 May 2017; Accepted 24 May 2017; Published 21 June 2017

Academic Editor: Antonina Pirrotta

Copyright (C) 2017 Zhenning Ba and Xi Gao. This is an open access article distributed under the Creative Commons Attribution License, which permits unrestricted use, distribution, and reproduction in any medium, provided the original work is properly cited.

\begin{abstract}
The dynamic soil-structure interaction (SSI) for incident plane SH waves is analyzed for a two-dimensional (2D) model of a shear wall on a rigid foundation by using the indirect boundary element method (IBEM). The rigid foundation utilized in this study is embedded in transversely isotropic (TI) soil layers over bedrock. The accuracy of the IBEM method is verified and analyzed by setting a semicylindrical, rigid foundation-shear wall structure system in the single TI soil layer and multiple TI soil layers over bedrock. This study shows that the TI characteristics of the site have a significant impact on the effective input motion and the superstructure response. In a single soil layer, the increase in the shear modulus ratio in the vertical and horizontal directions has a certain degree of amplified action on the effective input motion and the superstructure response. Simultaneously, the corresponding peak frequency of the response increases. In multiple soil layers, the changes in the effective input motion and the superstructure response are also affected by the TI characteristics of the soil layers, and the impact of this effect is related to the sequence of the layers.
\end{abstract}

\section{Introduction}

Dynamic soil-structure interactions (SSI) under the effect of seismic waves are important problems in the fields of soil dynamics, seismology, and earthquake engineering, because of their particular significance to practical engineering applications. Methods for solving soil-structure interaction problems can be divided into analytical and numerical methods. Early analytical methods utilized wave function expansion to obtain the out-of-plane response of a two-dimensional (2D), semicircular, rigid foundation (with a shear wall representing the superstructure) when excited by plane $\mathrm{SH}$ waves [1, 2]. Subsequently, many scholars adopted analytical methods to study dynamic soil-structure interactions, including the dynamic response of the interaction of a structure system with different foundations. Semielliptical and hemispherical foundations are some examples of differing foundations [36]. In addition, the interaction of a soil-structure system under different soil conditions (dry, saturated, etc.) was studied [7-9].

The analytical method is limited to the dynamic response of foundations with a single shape in a homogeneous halfspace. However, in recent years, the development of computer technology has promoted the application of numerical methods, such as the finite element method and the boundary element method. The key to study the dynamic interaction between soil and structure using the finite element method is to apply the appropriate artificial transmitting boundary. Lysmer [10] was the first to utilize the finite element method in this manner and calculated the dynamic response of a three-dimensional foundation embedded in a homogeneous half-space by setting a viscous absorbing boundary condition. Tassoulas and Kausel [11] calculated all of the stiffness coefficients of foundations embedded in a layered half-space using the semidiscrete method and setting a rigid transmitting boundary. They also discussed the influence of the 
embedded depth of the foundation in detail. Subsequently, many other scholars proposed other boundary conditions, including the rigid transmitting boundary [12], the iterative transmitting boundary [13], the Smith boundary [14], the paraxial boundary [15, 16], and the Higdon boundary [17]. These researchers conducted in-depth studies on the dynamic interactions between soil and structure.

The boundary element method (BEM) does not require artificial boundaries because it uses Green's functions as a basic solution, which automatically satisfies the radiation conditions at infinity. Green's functions have also been used to calculate the impedance functions of embedded foundations in [18]. Karabalis and Beskos $[19,20]$ studied the stiffness coefficients of three-dimensional and two-dimensional embedded foundations and their dynamic response under seismic action using the time integral method. de Barros and Luco [21] calculated the dynamic response of a twodimensional semicircular foundation embedded in a layered half-space using Green's function. Fu et al. [22-25] studied the dynamic response of the interaction of a two-dimensional, semicylindrical, rigid foundation-shear wall system in single and multiple soil layers using the boundary element method combined with Green's function distributed on a slope.

In order to simplify the model, most scholars assumed the subject site to be a homogenous or isotropic half-space, which was different from the actual site. Studies have shown that the soil in an actual site often is anisotropic in different degrees, and the anisotropy is mainly reflected in the difference in elastic properties in the horizontal and vertical directions. Therefore, it is more realistic to consider the ground soil as TI soil [26]. Compared to isotropic ground soil, there is much less research available on the dynamic interaction of a soil-structure in TI soil. In existing studies, Wang and Rajapakse [27] calculated the dynamic response of a twodimensional foundation under low and medium frequency using Green's function $[28,29]$ for anisotropic soil; Kirkner [30] studied the dynamic interaction of TI half-space and a circular foundation for the first time; and Wu et al. [31] used an analytical method to investigate the vibration problem of a rigid round plate on TI-saturated half-space under the action of harmonic torsional load. Although these studies have adopted the site model of a TI soil layer, they did not have access to a systematical analysis or research on the influence of the characteristic parameters of TI ground soil on the dynamic response of soil-structure.

In this study, the layered characteristic of natural soil has been considered, Green's function of distributed loads on inclined lines with the indirect boundary element method (IBEM) is adopted as an elementary solution, and the solution of the effective input motion and the superstructure response in the dynamic interaction of soil-structure in TI ground soil has been given. By comparing with the effective input motion and the superstructure response in isotropic ground soil, the method has been verified; the effective input motion and the superstructure response are calculated by setting the single TI soil layer and the multiple TI soil layers as examples. In addition, the influence of the TI characteristics of the ground

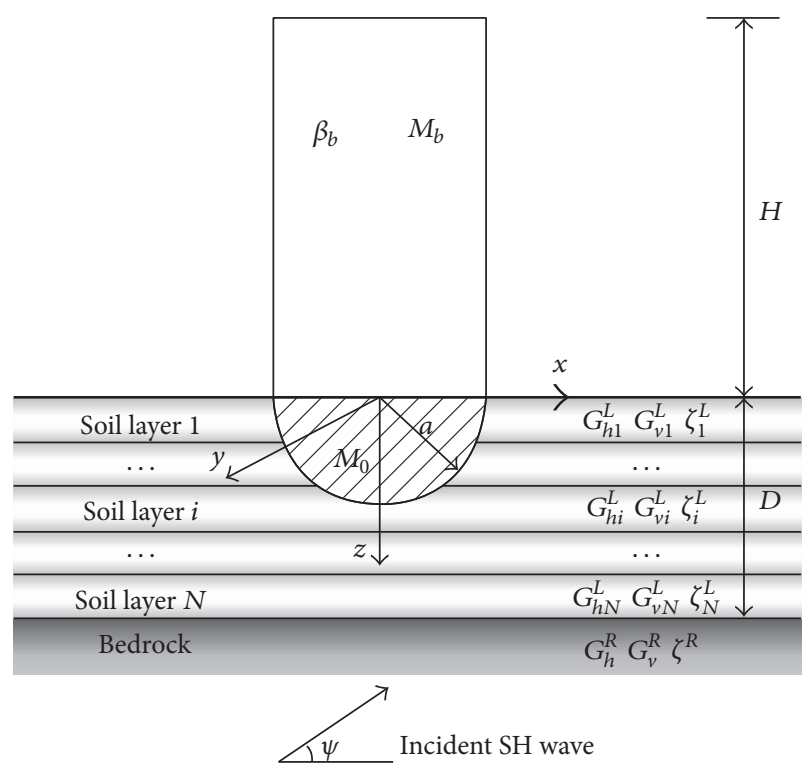

FIGURE 1: Foundation-shear wall model.

soil on the effective input motion and the superstructure response has been analyzed and discussed.

\section{Methodology}

2.1. Model. The model shown in Figure 1 is embedded in TI-layered ground soil and consists of a rigid semicylinder foundation that supports an elastic shear wall. The TI ground is formed using $N$ layers of horizontal TI soil and TI bedrock (half-space) underneath. In TI soil, the axis of symmetry of the medium is assumed to be normal to the horizontal surface. The TI soil layer is determined by the shear modulus in plane normal to axis of symmetry $G_{h i}^{L}$, horizontal shear modulus, the shear modulus in planes normal to plane of transverse isotropy $G_{v i}^{L}$, vertical shear modulus [32,33], and the damping ratio $\zeta_{i}^{L}(i=1 \sim N)$. The TI bedrock (halfspace) is determined by the shear modulus in the horizontal direction $G_{h}^{R}$; the shear modulus in the vertical direction $G_{v}^{R}$; and the damping ratio $\zeta^{R}$. The superscripts $L$ and $R$ represent the soil layer and the bedrock (half-space). The cross section of the foundation is semicircular, but it extends along the $y$ axis with radius of $a$ and a mass of $M_{0}$. The shear wall has the same width as the foundation with a shear wave velocity of $\beta_{b}$, a height of $H$, a mass of $M_{b}$ per unit length, and a damping ratio $\zeta^{b}$. The shear wall has a rigid connection (no slip) between the rigid foundation and the TI-layered ground soil, and the interface between the foundation and the ground soil is $S$. Incident $\mathrm{SH}$ wave inputs from angle $\psi$ in the bedrock surface have a circular frequency of $\omega$. In the case of incoming $\mathrm{SH}$ waves, the foundation response (i.e., the effective input motion) and the superstructure response are sought after.

The main steps for determining the foundation response (i.e., the effective input motion) and the superstructure response include (1) the direct stiffness method which was introduced in literature [34] is adopted to determine the 
response of the free field in TI ground soil; (2) the indirect boundary element method (IBEM) from literature [35] is used to determine the dynamic stiffness coefficient outside of the embedded foundation plane; (3) the effective input motion is solved for, in other words, the sum of the following two parts of the response which is determined: (a) the response when the mass $M_{0}$ of foundation and the mass $M_{b}$ of superstructure are not taken into account; (b) the additional displacement response when the mass inertia forces $F_{0}$ and $F_{b}$ of the foundation and superstructure are taken into account; (4) the method in [2] is used to determine the superstructure response; (5) the relative effective input motion and superstructure response with respect to the free field are determined, namely, the specific value of the displacement amplitude of that point before and after the existence of superstructure.

\subsection{The Response of the Free Field in Layered TI Ground} Soil. The response of the free field refers to the site response when there is no foundation or superstructure. Based on the accurate out-of-plane dynamic stiffness matrix for TI-layered medium given in the literature [36], the direct stiffness method introduced in literature [34] is adopted to determine the response of the free field in TI ground soil. For the stiffness matrix of the TI soil layer, we assume that there are ascending and descending $\mathrm{SH}$ waves in the layer, and then the displacement at any point in the layer can be expressed as

$$
\begin{aligned}
v(x, z) \\
\quad=\left[A_{\mathrm{SH}} \exp (i k t z)+B_{\mathrm{SH}} \exp (-i k t z)\right] \exp (-i k x) \\
=v(z) \exp (-i k x),
\end{aligned}
$$

where $v(z)$ can be considered as the amplitude transmitting along the $x$-direction

$$
v(z)=A_{\mathrm{SH}} \exp (i k t z)+B_{\mathrm{SH}} \exp (-i k t z)
$$

where $A_{\mathrm{SH}}$ and $B_{\mathrm{SH}}$ are the amplitudes of the ascending and descending $\mathrm{SH}$ waves in the soil layer, respectively, $k=$ $\omega \cos \psi_{\mathrm{SH}}^{L} / c_{s}^{L *}$ is the wave number along the $x$-direction, and $t=-i \sqrt{1-1 / \cos ^{2} \psi_{\mathrm{SH}}^{L}}$ and $\psi_{\mathrm{SH}}^{L}$ are the included angles of the ascending or descending $\mathrm{SH}$ waves in the soil layer in the horizontal direction

$$
c_{s}^{L *}=\sqrt{\left(c_{s h}^{L *}\right)^{2} \cos ^{2} \psi_{S H}^{L}+\left(c_{s v}^{L *}\right)^{2} \sin ^{2} \psi_{S H}^{L}},
$$

which is the complex wave velocity of SH waves transmitting in the $\psi_{\mathrm{SH}}^{L}$ direction. $c_{s h}^{L *}=\sqrt{G_{h}^{L *} / \rho}$ and $c_{s v}^{L *}=\sqrt{G_{v}^{L *} / \rho}$ are the complex wave velocities of SH waves in a soil layer transmitting in the horizontal and vertical directions, $G_{h}^{L *}=$ $G_{h}^{L}\left(1+2 i \zeta^{L}\right)$ and $G_{v}^{L *}=G_{v}^{L}\left(1+2 i \zeta^{L}\right)$ are the complex shear moduli in the horizontal and vertical directions, $G_{h}^{L}$ and $G_{v}^{L}$ are the horizontal and vertical shear moduli when there is no damping in the soil layer, and $\zeta^{L}$ is the damping ratio.
By substituting $z=0$ and $z=d$ ( $d$ is the thickness of soil layer) into (2), the displacement amplitudes $v_{1}$ and $v_{2}$ of the top and bottom of the soil layer can be obtained as

$$
\left\{\begin{array}{l}
v_{1} \\
v_{2}
\end{array}\right\}=\left[\begin{array}{cc}
1 & 1 \\
\exp (i k t d) & \exp (-i k t d)
\end{array}\right]\left\{\begin{array}{l}
A_{\mathrm{SH}} \\
B_{\mathrm{SH}}
\end{array}\right\} .
$$

Through the relational expression of stress and displacement, the shear stresses $\tau_{y x}$ and $\tau_{y z}$ can be obtained as

$$
\begin{aligned}
\tau_{y x} & =G_{h}^{L *} \frac{\partial v}{\partial x} \\
& =i k t G_{h}^{L *}\left[A_{\mathrm{SH}} \exp (i k t z)-B_{\mathrm{SH}} \exp (-i k t z)\right] \\
& \cdot \exp (-i k x), \\
\tau_{y z} & =G_{v}^{L *} \frac{\partial v}{\partial z} \\
& =i k t G_{v}^{L *}\left[A_{\mathrm{SH}} \exp (i k t z)-B_{\mathrm{SH}} \exp (-i k t z)\right] \\
& \cdot \exp (-i k x) .
\end{aligned}
$$

By substituting $z=0$ and $z=d$ into (6) and introducing the amplitudes of the external load, $Q_{1}=\tau_{y z}(0)$ and $Q_{2}=$ $\tau_{y z}(d)$, the amplitudes of the external load on the top and at the bottom of the soil layer can be obtained as

$$
\left\{\begin{array}{l}
Q_{1} \\
Q_{2}
\end{array}\right\}=i k t G_{v}^{L *}\left[\begin{array}{cc}
-1 & 1 \\
\exp (i k t d) & -\exp (-i k t d)
\end{array}\right]\left\{\begin{array}{l}
A_{\mathrm{SH}} \\
B_{\mathrm{SH}}
\end{array}\right\} .
$$

Eliminating coefficients $A_{\mathrm{SH}}$ and $B_{\mathrm{SH}}$ in (4) and (7), the layered stiffness matrix $\left[S_{\mathrm{SH}}^{L}\right]$ of the TI soil layer is

$$
\begin{gathered}
\left\{\begin{array}{l}
Q_{1} \\
Q_{2}
\end{array}\right\}=\left[\begin{array}{l}
\left.S_{\mathrm{SH}}^{L}\right] \\
v_{1} \\
v_{2}
\end{array}\right\}=\frac{\omega \sin \psi_{\mathrm{SH}}^{L} G_{v}^{L *}}{c_{s}^{L *} \sin \left(\omega \sin \psi_{\mathrm{SH}}^{L} / c_{s}^{L *}\right)} \\
\cdot\left[\begin{array}{cc}
\cos \left(\frac{\omega \sin \psi_{\mathrm{SH}}^{L}}{c_{s}^{L *}} d\right) & -1 \\
-1 & \cos \left(\frac{\omega \sin \psi_{\mathrm{SH}}^{L}}{c_{s}^{L *}} d\right)
\end{array}\right]\left\{\begin{array}{c}
v_{1} \\
v_{2}
\end{array}\right\} .
\end{gathered}
$$

Under an infinite radiation condition, the outgoing wave with an amplitude of $B_{\mathrm{SH}}$ will be generated if load is arranged on the surface of TI half-space. The subscript 0 represents the free surface of the TI half-space in (4) and (7). Setting $A_{\mathrm{SH}}=$ $0, v_{0}=v_{1}$, and $Q_{0}=Q_{1}=-\tau_{y z 0}$, the stiffness coefficient $S_{\mathrm{SH}}^{R}$ of the TI half-space will be obtained after the elimination of $B_{\mathrm{SH}}$

$$
Q_{0}=S_{S H}^{R} v_{0}=i G_{v}^{R *} \frac{\omega \sin \psi_{S H}^{R}}{c_{s}^{R *}} v_{0} .
$$

After determining all of the stiffness matrices in the TI soil layer and the TI half-space, the accurate dynamic stiffness matrix $\left[S_{\mathrm{SH}}\right]$ of the TI-layered site can be obtained, by combining $\left[S_{\mathrm{SH}}^{L}\right]$ and $S_{\mathrm{SH}}^{R}$ 


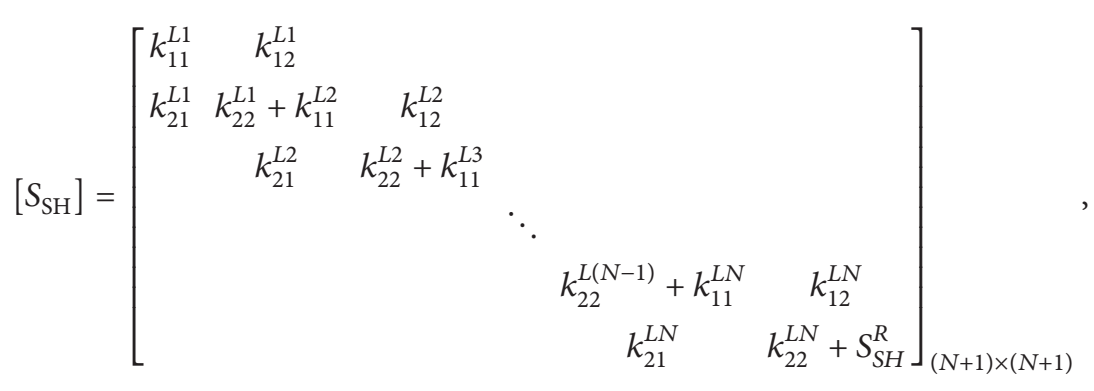

where $k_{i j}^{L n}(n=1 \sim N, i j=1 \sim 2$ ) denotes the $i j$ th submatrix of the $n$th TI layer's dynamic stiffness matrix $\left[S_{\mathrm{SH}}^{L}\right]$. Therefore, the discrete dynamic equilibrium equation in the TI-layered site can be expressed as

$$
\left\{Q_{1}, Q_{2}, \ldots, Q_{n}\right\}^{T}=\left[S_{\mathrm{SH}}\right]\left\{v_{1}, v_{2}, \ldots, v_{n}\right\}^{T},
$$

where $\left\{Q_{1}, Q_{2}, \ldots, Q_{n}\right\}^{T}$ is the vector of the amplitude of an external load acting on the interface of the soil layer and $\left\{v_{1}, v_{2}, \ldots, v_{n}\right\}^{T}$ is the vector of the displacement amplitude on the interface of the soil layer.

After obtaining the overall dynamic stiffness matrix of TI-layered site, the control point for SH wave transmitting from the bedrock surface can be selected on the outcrop of the bedrock, so that the other elements are zero in the vector $\left\{Q_{1}, Q_{2}, \ldots, Q_{n}\right\}^{T}$ of amplitude of external load, only

$$
Q_{n}=S_{\mathrm{SH}}^{R} v_{0}=i G_{v}^{R *} \frac{\omega \sin \psi_{\mathrm{SH}}^{R}}{c_{s}^{R *}} v_{0},
$$

where $G_{v}^{R *}$ is the complex shear modulus of the bedrock halfspace in the vertical direction, $\psi_{\mathrm{SH}}^{R}$ is the incident angle of $\mathrm{SH}$ waves in the bedrock half-space, and $c_{s}^{R *}$ is the complex wave velocity of $\mathrm{SH}$ waves of the bedrock transmitting in the incident direction. In (3), $v_{0}$ represents the movement of the bedrock outcrop. Substituting (12) into (11), the displacement in the interface of the TI soil layer can be obtained, and the amplitude coefficient of the ascending and descending wave in any soil layer can be obtained using (4). Finally, the amplitude of displacement and stress in arbitrary point of the TI-layered half-space can be obtained using (1), (5), and (6), namely, the free field response of $\mathrm{SH}$ waves.

\subsection{Green's Function of the Uniformly Distributed Inclined} Load Acting in the TI Medium. In this section, the Green's function of the uniformly distributed inclined load acting in the TI medium is derived, including both the displacement and stress, which serve the elementary solution of IBEM to obtain the stiffness coefficient of the foundation. The specific deviation of Green's function is described as follows.

Firstly, the load uniformly distributed on inclined lines in the spatial domain is expanded to the wave number domain. Secondly, two fictitious interfaces are introduced at the upper and lower boundaries of the loaded layer. In order to derive the dynamic response of the loaded layer, the two fictitious interfaces have to be fixed to obtain the counterforces of the two ends. Actually, the dynamic response of the loaded layer includes the particular solutions induced by the load applied, homogeneous solutions caused by the reaction loads and the solution derived from the reverse counterforces at the two ends. However, for those unloaded TI layers, their solutions only contain those homogeneous ones induced by the reverse counterforces at the two ends of the loaded layer. Finally, the solutions for every TI layer in the spatial domain are obtained by superposing a Fourier inversion on those solutions in the wave number domain, that is, Green's function of uniformly distributed load on inclined lines [37]. Green's function of displacement and tractive force (assuming that normal direction is known) is presented below:

$$
\begin{aligned}
g_{u} & \left(\mathbf{x}, \xi_{l}\right) \\
& =\int_{-\infty}^{\infty}\left[g_{u}^{p}\left(\mathbf{x}, \xi_{l}, k\right)+g_{u}^{h}\left(\mathbf{x}, \xi_{l}, k\right)+g_{u}^{r}\left(\mathbf{x}, \xi_{l}, k\right)\right] \\
& \cdot e^{-i k x} d k, \\
g_{t} & \left(\mathbf{x}, \xi_{l}\right) \\
& =\int_{-\infty}^{\infty}\left[g_{t}^{p}\left(\mathbf{x}, \xi_{l}, k\right)+g_{t}^{h}\left(\mathbf{x}, \xi_{l}, k\right)+g_{t}^{r}\left(\mathbf{x}, \xi_{l}, k\right)\right] \\
& \cdot e^{-i k x} d k,
\end{aligned}
$$

and Green's function of displacement and tractive force for those unloaded TI layers is described as follows:

$$
\begin{aligned}
& g_{u}\left(\mathbf{x}, \xi_{l}\right)=\int_{-\infty}^{\infty} g_{u}^{r}\left(\mathbf{x}, \xi_{l}, k\right) e^{-i k x} d k, \\
& g_{t}\left(\mathbf{x}, \xi_{l}\right)=\int_{-\infty}^{\infty} g_{t}^{r}\left(\mathbf{x}, \xi_{l}, k\right) e^{-i k x} d k,
\end{aligned}
$$

where $g_{u}\left(\mathbf{x}, \xi_{l}\right)$ and $g_{t}\left(\mathbf{x}, \xi_{l}\right)$ are Green's functions of displacement and tractive force for distributed loads. This applies when the loads are distributed on inclined lines with density $q\left(\xi_{l}\right)$ in the unit (midpoint $\xi_{l}$ ). The superscript " $p$ " represents the particular solution in the fixed layer, " $h$ " the homogeneous solution in the fixed layer, " $r$ " the solution to the counterforce in fixed end, and " $k$ " the wave number along the horizontal direction. 
After determining Green's function, the total displacement and tractive force of any point $\mathbf{x}=(x, z)$ in the TIlayered ground soil can be expressed as

$$
\begin{aligned}
v(\mathbf{x}) & =\sum_{l=1}^{K} g_{u}\left(\mathbf{x}, \xi_{l}\right) q\left(\xi_{l}\right), \\
t_{y}(\mathbf{x}) & =\sum_{l=1}^{K} g_{t}\left(\mathbf{x}, \xi_{l}\right) q\left(\xi_{l}\right),
\end{aligned}
$$

where $K$ is the total unit number. The total number of units (element size) is determined by the wavelength of the elastic wave in the TI-layered ground soil. This is necessary in order to meet the requirement of discrete convergence. Equations (15) and (16) can be understood as the dynamic response (scattered wave field) inside the layered TI halfspace that is generated by the movement of the foundation. In addition, they can be simulated as the total dynamic response generated by applying a uniformly distributed load on all of the boundary units of the foundation.

2.4. Out-of-Plane Dynamic Stiffness Coefficient. Because the connection between the ground soil and the foundation is completely rigid, the displacements of the points in boundary $S$ of the foundation are the same. This displacement is equal to the out-of-plane displacement $\Delta$ of the rigid foundation (the foundation can only generate out-of-plane displacement under the excitation of out-of-plane load), and the displacements of points in the boundary $S$ of the foundation can be expressed as

$$
v(\mathbf{x})=\sum_{l=1}^{K} g_{u}\left(\mathbf{x}, \xi_{l}\right) q\left(\xi_{l}\right)=\Delta .
$$

Equation (17) can also be written as

$$
\sum_{l=1}^{K} g_{u}\left(\mathbf{x}, \xi_{l}\right) \frac{q\left(\xi_{l}\right)}{\Delta}=1=\sum_{l=1}^{N} g_{u}\left(\mathbf{x}, \xi_{l}\right) \Lambda_{l},
$$

where $\Lambda_{l}(l=1 \sim K)$ represents the density of the uniformly distributed load applied on the $l$ th unit when the foundation has unit displacement. Assuming that the rigid contact boundary conditions in $S$ of the foundation can be independently met at $\xi_{l}$ of each unit, an equation set formed by $K$ equations about $\Lambda_{1}, \Lambda_{2}, \ldots, \Lambda_{K}$ will be obtained by combining (18). $\Lambda_{l}(l=1 \sim K)$ can be obtained by solving the linear system of equations. The following will be obtained by substituting $\Lambda_{l}$ into (16)

$$
t_{y}(\mathbf{x})=\sum_{l=1}^{K} g_{t}\left(\mathbf{x}, \xi_{l}\right) \Lambda_{l} \Delta
$$

The resultant force acting on the foundation will be determined by integrating (19) along the boundary

$$
F_{y}=\int_{S} t(\mathbf{x}) d s=\int_{S} \sum_{l=1}^{K} g_{t}\left(\mathbf{x}, \xi_{l}\right) \Lambda_{l} \Delta d s=K_{y y} \Delta .
$$

Equation (20) represents the relational expression of force and displacement acting on the foundation, while $K_{y y}$ is the dynamic stiffness coefficient of the foundation

$$
K_{y y}=\int_{S} \sum_{l=1}^{K} g_{t}\left(\mathbf{x}, \xi_{l}\right) \Lambda_{l} d s
$$

2.5. The Effective Input Motion and the Superstructure Response. The effective input motion $\Delta$ is the displacement response of the foundation under harmonic wave excitation. The displacement response $\Delta$ includes two parts $\Delta_{1}$ and $\Delta_{2}$ : the first one is the response without considering the mass $M_{0}$ of the foundation and the mass $M_{b}$ of the superstructure and the second one is the additional displacement response that considers the mass inertia force $F_{0}$ of the foundation and the mass inertia force $F_{b}$ of the superstructure. In the first part above, the virtual work principle is applied to the problem of displacement field and stress field of scattering and radiation of incident wave to obtain the displacement response $\Delta_{1}$ which has been used in [38]. The displacement response $\Delta_{1}$ is applied to the embedded foundation in [39], identified as

$$
\Delta_{1}=\frac{\int_{S}\left[v_{f}(x, z) \sum_{l=1}^{K} g_{t}\left(\mathbf{x}, \xi_{l}\right) \Lambda_{l}-t_{f}\left(\mathbf{x}, \xi_{l}\right)\right] d s}{K_{y y}},
$$

in which $v_{f}$ and $t_{f}$ are the out-of-plane displacement and stress of the free field, respectively, along $S$.

The displacement response in the second part, $\Delta_{2}$, can be obtained using

$$
\Delta_{2}=\frac{F_{0}+F_{b}}{K_{y y}} .
$$

The foundation is a rigid body and the following can be obtained under dynamic load

$$
F_{b}=\omega^{2} M_{0} \Delta .
$$

The shear wall is an elastomer and the inertial force can be obtained by elastic dynamics

$$
F_{b}=\omega^{2} M_{b} \frac{\tan k_{b} H}{k_{b} H} \Delta,
$$

in which $k_{b}=\omega / \beta_{b}$ represents the shear wave number of the shear wall.

Substituting (23), (24), and (25) into $\Delta=\Delta_{1}+\Delta_{2}$, the effective input motion can be obtained

$$
\Delta=\frac{\Delta_{1}}{1-\left(\omega^{2} / K_{y y}\right)\left(M_{0}+M_{b}\left(\tan k_{b} H / K_{b} H\right)\right)} .
$$

The superstructure response $\Delta_{b}$ is the relative displacement between the top of the shear wall and the foundation and can be obtained from [10]

$$
\Delta_{b}=\Delta\left(\frac{1}{\cos k_{b} H}-1\right) .
$$




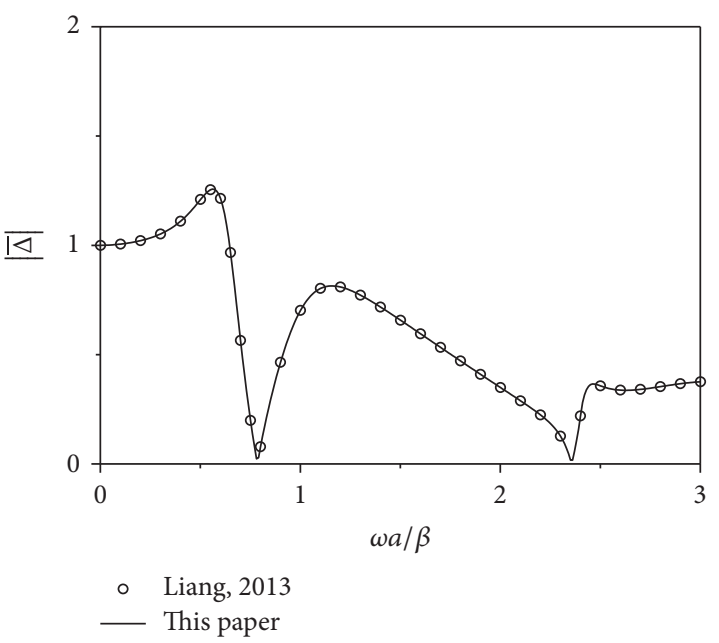

(a)

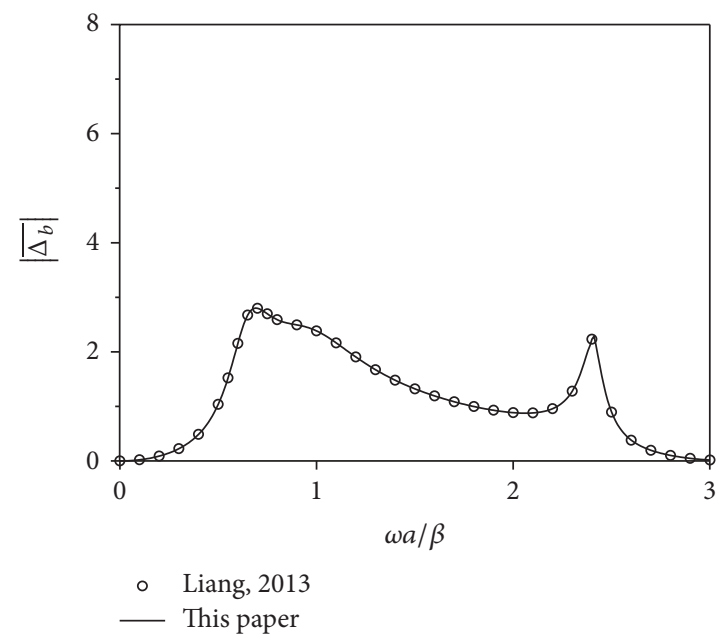

(b)

FIgURE 2: Comparison between study results and literature results [23].

The relative effective input motion and superstructure response with respect to the free field (i.e., the specific value of the displacement amplitude of that point before and after the existence of the superstructure) can be represented by the following:

$$
\begin{aligned}
\bar{\Delta} & =\frac{\Delta}{\left|v_{f}\right|}, \\
\bar{\Delta}_{b} & =\frac{\Delta_{b}}{\left|v_{f}\right|} .
\end{aligned}
$$

\section{Verification of Accuracy}

The method used in this study is verified through comparison with the effective input motion of isotropic media and the superstructure response given in $[2,23]$. According to our method, the results in isotropic ground soil can be calculated by using the same shear modulus in the horizontal and vertical directions $\left(G_{v}=G_{h}\right)$. In order to describe the stiffness of the shear wall, a dimensionless parameter, $\varepsilon=\beta_{L} H / \beta_{b} a$, is defined in which $H$ and $\beta_{b}$ are the parameters of the shear wall. We set $\beta_{R} / \beta_{L}=2, \varepsilon=2$ in Figures 2 and 3 to perform the numerical calculation and the results obtained in this study are compared to existing ones. It can be seen from the figures that our results correlate well with the results obtained by Trifunac [2] and Liang et al. [23].

\section{Numerical Results and Analysis}

4.1. Single TI Soil Layer. Taking the semicylindrical rigid foundation embedded in single TI soil layer on isotropic bedrock as an example, the foundation extends infinitely in the $y$-axis direction with the same cross section. The superstructure is simplified to be a piece of shear wall with the same width, and there is a rigid connection between the rigid

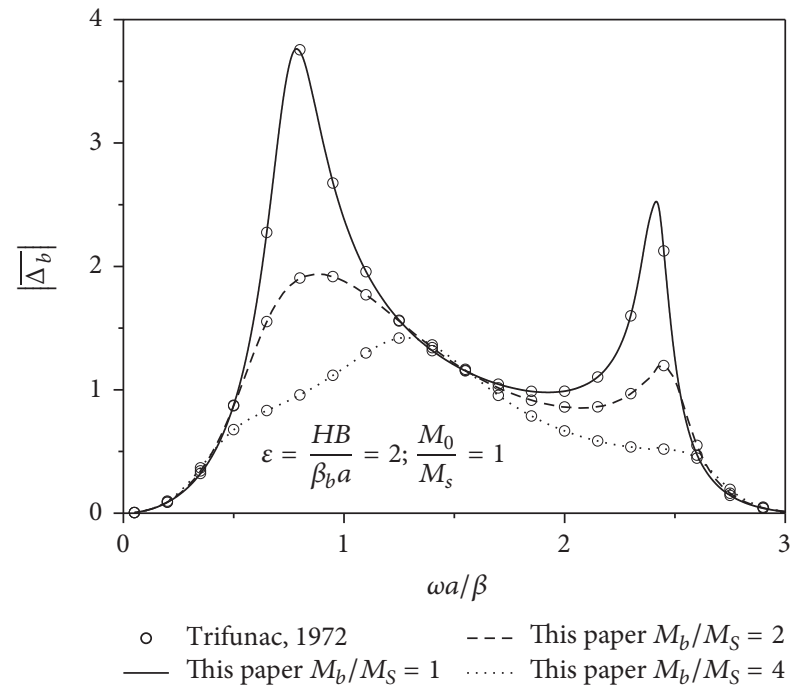

Figure 3: Comparison between study results and literature results [2].

foundation and TI soil (no slip). Figures 4, 5, 6, and 7 show the effective input motion and the superstructure response when the shear modulus ratio in the vertical and horizontal directions of TI soil is different. During the calculation, the shear modulus ratio is taken as $G_{v} / G_{h}=0.5,1.0$, and 2.0. The bedrock is considered to be isotropic with a density ratio of $\rho^{R} / \rho^{L}=1.0$, and the damping ratio of the soil layer and bedrock is $\zeta^{L}=0.05$ and $\zeta^{R}=0.02$, respectively. The thickness of TI soil layers is $D / a=2,3,4,5$, the mass of the upper shear wall is $M_{b} / M_{0}=1,2,4$, and the rigid of the shear wall is $\varepsilon=0,2,4$. To maintain consistency in the vibration frequency of the soil under three different situations, the dimensionless frequency is still defined as $\omega a / \sqrt{\left(G_{V}+G_{h}\right) / 2 \rho}$. 

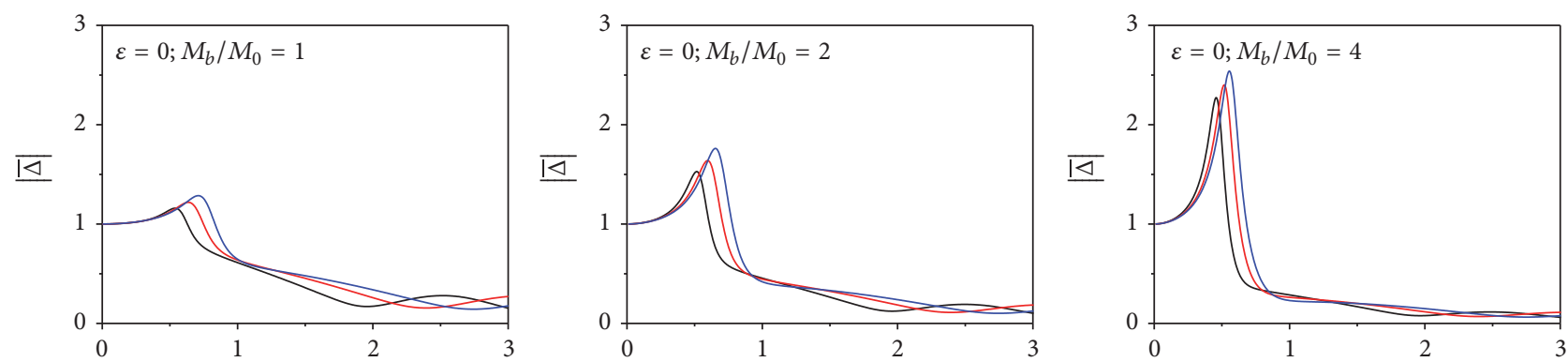
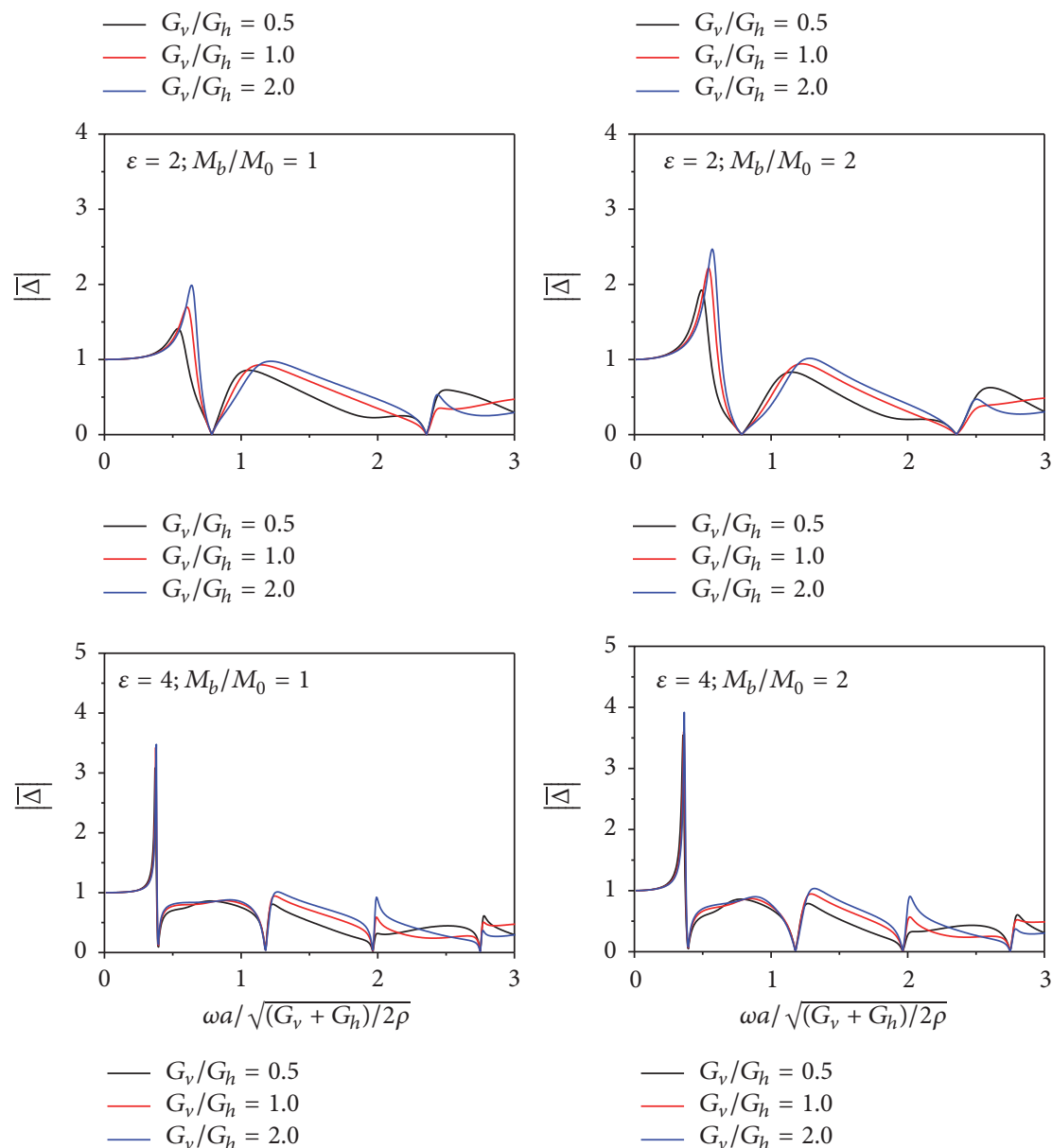
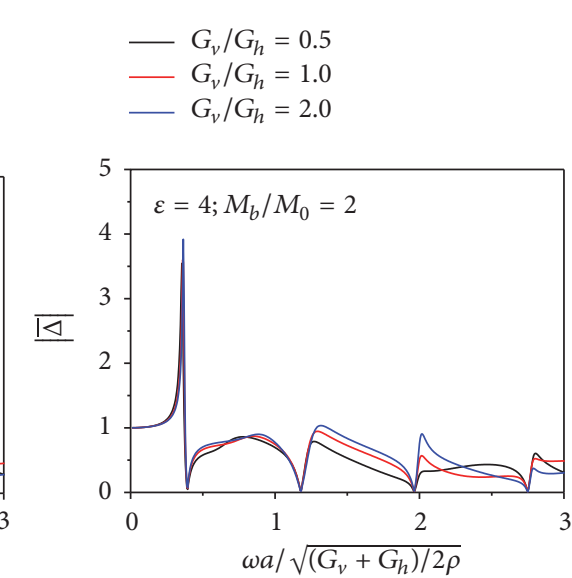

$\begin{aligned}-G_{v} / G_{h} & =0.5 \\ -G_{v} / G_{h} & =1.0 \\ -G_{v} / G_{h} & =2.0\end{aligned}$
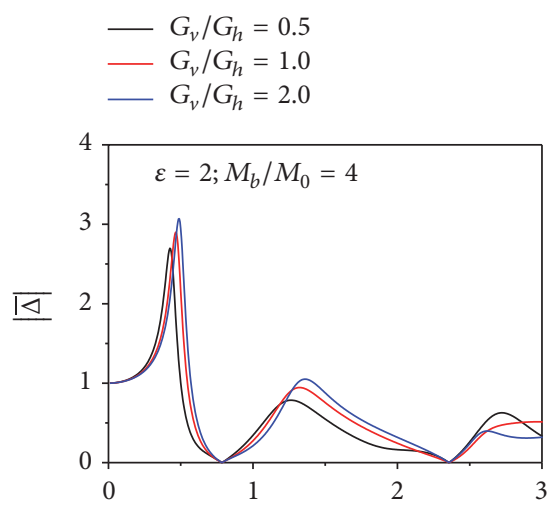

$$
\begin{aligned}
-G_{v} / G_{h} & =0.5 \\
-G_{v} / G_{h} & =1.0 \\
G_{v} / G_{h} & =2.0
\end{aligned}
$$

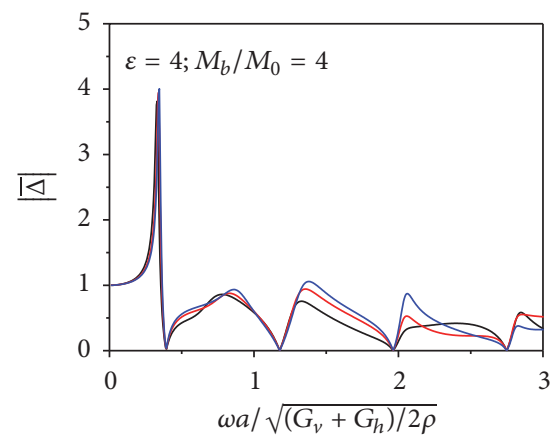

$\begin{aligned}-G_{v} / G_{h} & =0.5 \\ -G_{v} / G_{h} & =1.0 \\ -G_{v} / G_{h} & =2.0\end{aligned}$

FIGURE 4: The effective input motion for different flexibility and mass of superstructure and for $D / a=2$.

4.1.1. The Effective Input Motion. As shown in Figure 4, it can be found that the effective input motion $|\bar{\Delta}|$ in single TI soil layer $\left(G_{v} / G_{h}=0.5\right.$ and 2.0$)$ is completely different from that in single isotropic layer $\left(G_{v} / G_{h}=1.0\right)$ over bedrock. The effective input motion in single TI soil layer fluctuates along with the changing effective input motion in single isotropic layer, and an oscillation phenomenon occurs. According to [23], for $\varepsilon=$ 2 and $D / a=2$, the resonant frequencies $\omega a / \sqrt{\left(G_{V}+G_{h}\right) / 2 \rho}$ of the shear wall are $0.785,2.356,3.927, \ldots$; for $\varepsilon=4$ and $D / a=2$, the resonant frequencies $\omega a / \sqrt{\left(G_{V}+G_{h}\right) / 2 \rho}$ of the shear wall are $0.393,1.178,1.964, \ldots$; and so on. As the flexibility $(\varepsilon)$ of the shear wall increases, the resonant frequency points of the shear wall concentrate gradually.
The effective input motion at a resonant frequency point of the shear wall is zero, and it reaches its maximum value at the first inherent frequency point of the soil layer. This is consistent with research on the effective input motion of a semicylindrical embedded foundation in [2]. In addition, with the increase in $\varepsilon$, the peak value of the effective input motion increases and the corresponding frequency of its peak point decreases, and the peak value tends to concentrate at the same frequency point. Some numerical results for $D / a=2$ and $M_{b} / M_{0}=1$ are selected as shown in Table 1 . In Table 1, "first frequency" is the first peak frequency of effective input motion and "peak value" is the corresponding peak value.

Meanwhile, with the increase in the shear modulus ratio of the soil layer in the vertical and horizontal directions, 

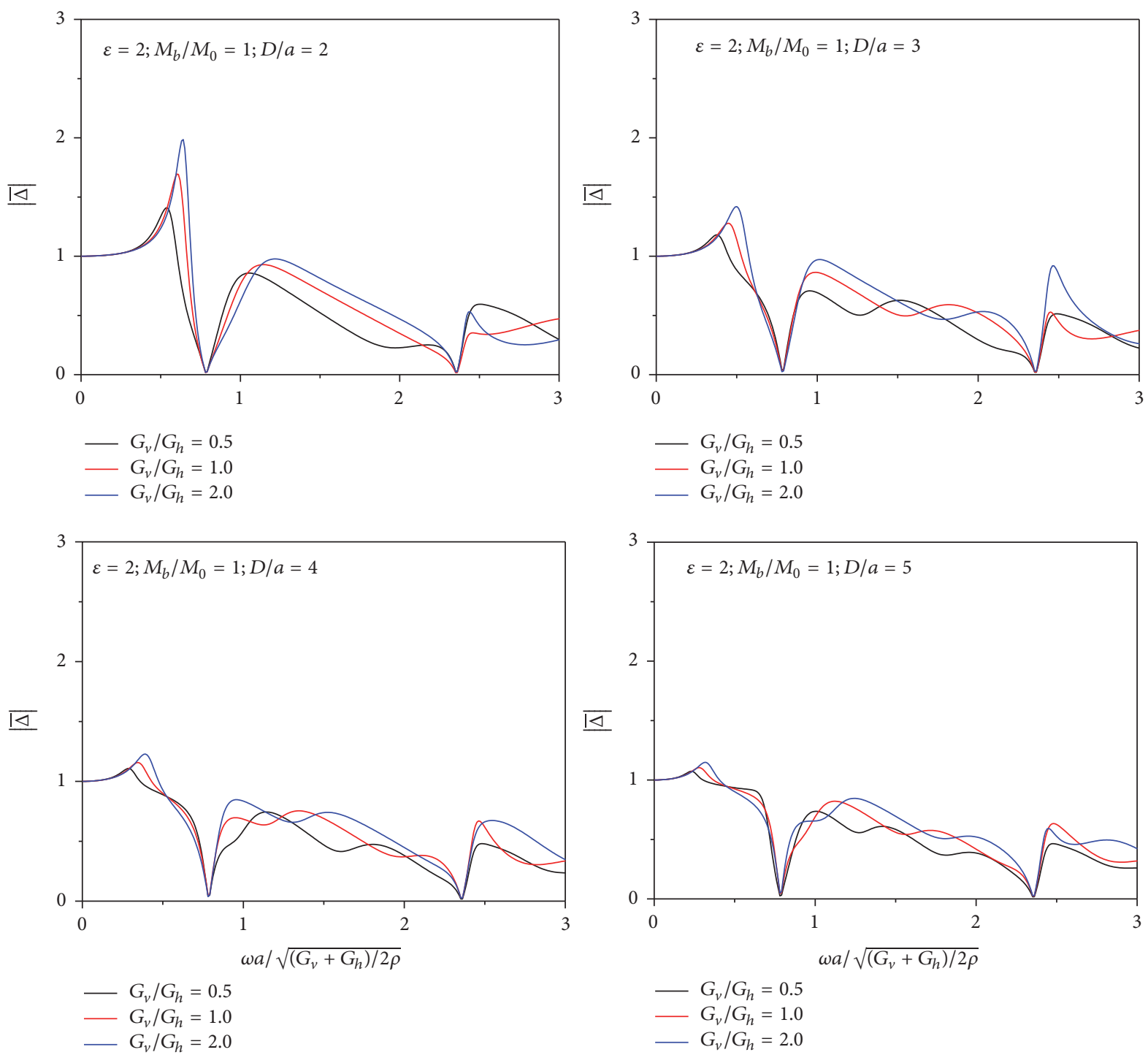

FIGURE 5: The effective input motion for different thicknesses of soil layer.

the corresponding frequency of peak value of the effective input motion tends to be higher. This trend is identical to the research on the resonance characteristics of SH waves traveling to a TI-layered site [40]. In addition, the corresponding frequency of the peak value of the effective input motion moves to a higher frequency as the shear modulus ratio in the vertical and horizontal directions increases. In addition, the peak value of the effective input motion also increases, which illustrates that the increase in the shear modulus ratio of the TI soil layer in the vertical and horizontal directions enlarges the response of the effective input motion. For example, for $\varepsilon=2, D / a=2, M_{b} / M_{0}=2$, and $G_{v} / G_{h}=0.5,1.0$, and 2.0 , the first peak value of effective input motion is 1.928 , 2.216, and 2.470. Moreover, with an increase in the mass of the upper shear wall structure, the peak value of the effective input motion increases gradually, and the maximum peak value appears at the first inherent frequency point of the TI soil layer. At this time, the parameters of the TI soil layer also have a significant impact on the effective input motion. With the increase in shear modulus in the vertical and horizontal directions, the peak value of the effective input motion soars up and the corresponding frequency moves to be higher. The author believes that the change in the parameters of the TI soil layer caused the change in the inherent frequency of the TI soil layer, which further leads to the peak change in the effective input motion.

In addition, as the thickness of the TI soil layer changes $(D / a=2,3,4$, and 5$)$, the effective input motion fluctuates in the frequency domain. When the thickness of the soil layer increases, the effective input motion at the resonant frequency point of the shear wall is still zero, but the peak value at the inherent frequency point of the soil layer decreases significantly, and its value tends to be the same. In summary, the greater the thickness of the TI soil layer is, the more significant the fluctuation of the effective input motion will be. Furthermore, as the shear modulus ratio of the soil 

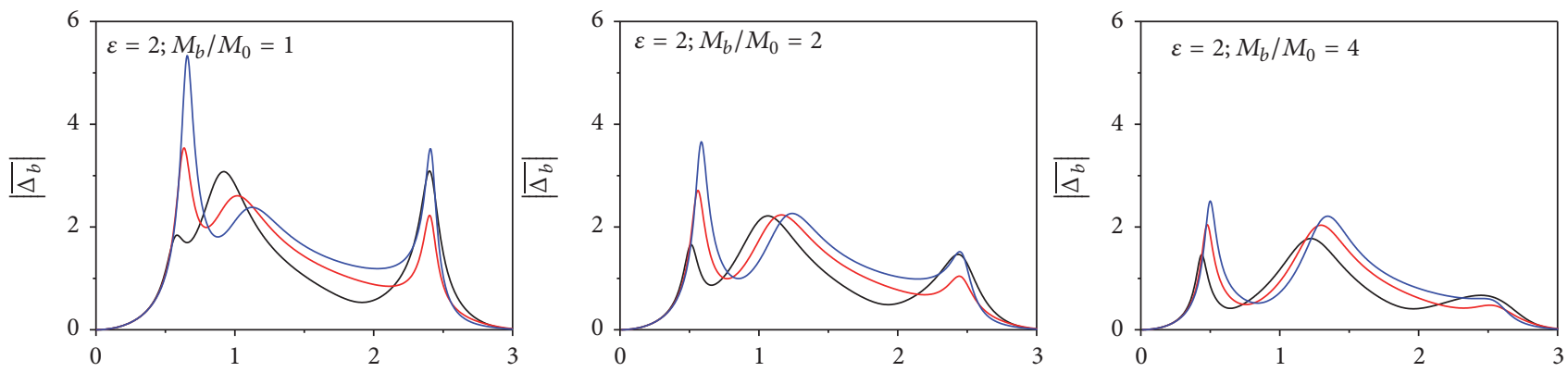
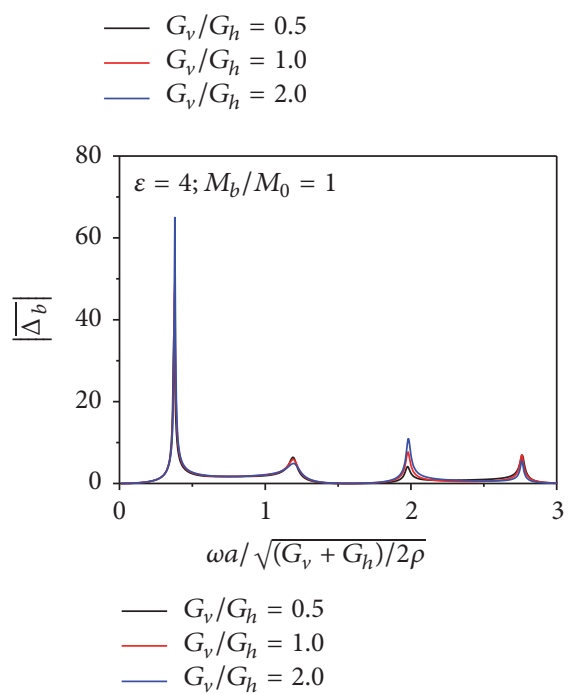

$\begin{aligned}-G_{v} / G_{h} & =0.5 \\ -G_{v} / G_{h} & =1.0 \\ -G_{v} / G_{h} & =2.0\end{aligned}$

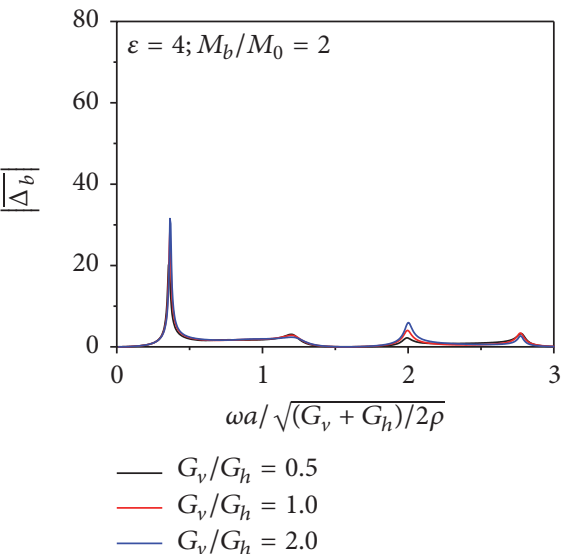

$-G_{v} / G_{h}=0.5$

$-G_{v} / G_{h}=1.0$

$-G_{v} / G_{h}=2.0$

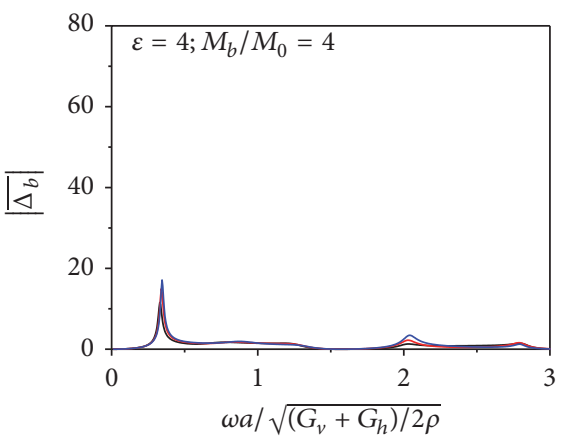

- $G_{v} / G_{h}=0.5$

$-G_{v} / G_{h}=1.0$

$-G_{v} / G_{h}=2.0$

FIgURE 6: The superstructure response for different flexibility and mass of superstructure and for $D / a=2$.

layer in the vertical and horizontal directions increases, the corresponding frequency of peak point of the effective input motion still moves to be higher. For example, for $\varepsilon=2$, $D / a=1, M_{b} / M_{0}=2$, and $G_{v} / G_{h}=0.5,1.0$, and 2.0 , the first peak frequency of effective input motion is $0.536,0.606$, and 0.636 .

4.1.2. The Superstructure Response. As shown in Figure 6, the superstructure response $\left|\bar{\Delta}_{b}\right|$ in single TI soil layer $\left(G_{v} / G_{h}=\right.$ 0.5 and 2.0) that can be found via calculation is quite different from that of the superstructure in single isotropic soil layer $\left(G_{v} / G_{h}=1.0\right)$. In addition, there is a certain vibration phenomenon that occurs in the low frequency range. The impact of soil TI parameters on the superstructure response in single TI soil layer is obviously greater than that in single isotropic soil layer. There are significant differences between the corresponding structural responses of foundations with different TI parameters, and the impact mainly occurs in the low frequency range. As shown in Figure 6, as the shear modulus ratio of the TI soil layer rises in the vertical and horizontal directions, the corresponding frequency of the peak point of the superstructure response is enhanced. Meanwhile, the value of the peak point gradually increases, which illustrates that the increase in the shear modulus ratio in the vertical and horizontal directions of the TI soil layer increases the response of the superstructure. For example, for $\varepsilon=2, D / a=4, M_{b} / M_{0}=2$, and $G_{v} / G_{h}=0.5,1.0$, and 2.0, the first peak value of the superstructure response is $1.460,2.045$, and 2.506 . In addition, when an increasing frequency, the impact of the parameters of the TI medium on the superstructure response weakens but tends to stabilize in the end.

The characteristics of the shear wall also have an influence on its relative displacement with respect to the foundation. As the mass of the upper shear wall $M_{b}$ increases, the effective input motion increases gradually, but the superstructure response weakens significantly. As a result, the corresponding frequency of its peak point augments. Therefore, it is clear that a change in the mass of the superstructure will lead to a change in the seismic energy distribution between the foundation and the superstructure. In addition, the superstructure response decreases when the effective input motion increases. Also, the flexibility $(\varepsilon)$ of the shear wall itself has a significant impact on the superstructure response. As the flexibility of the shear wall increases, the peak value of the superstructure response significantly increases, and the corresponding frequency of its peak point decreases. Moreover, as the thickness of the TI soil layer changes, the superstructure response fluctuates violently.

4.2. Multiple TI Soil Layers. Utilizing a semicylindrical foundation (embedded in TI soil) on an isotropic bedrock as 

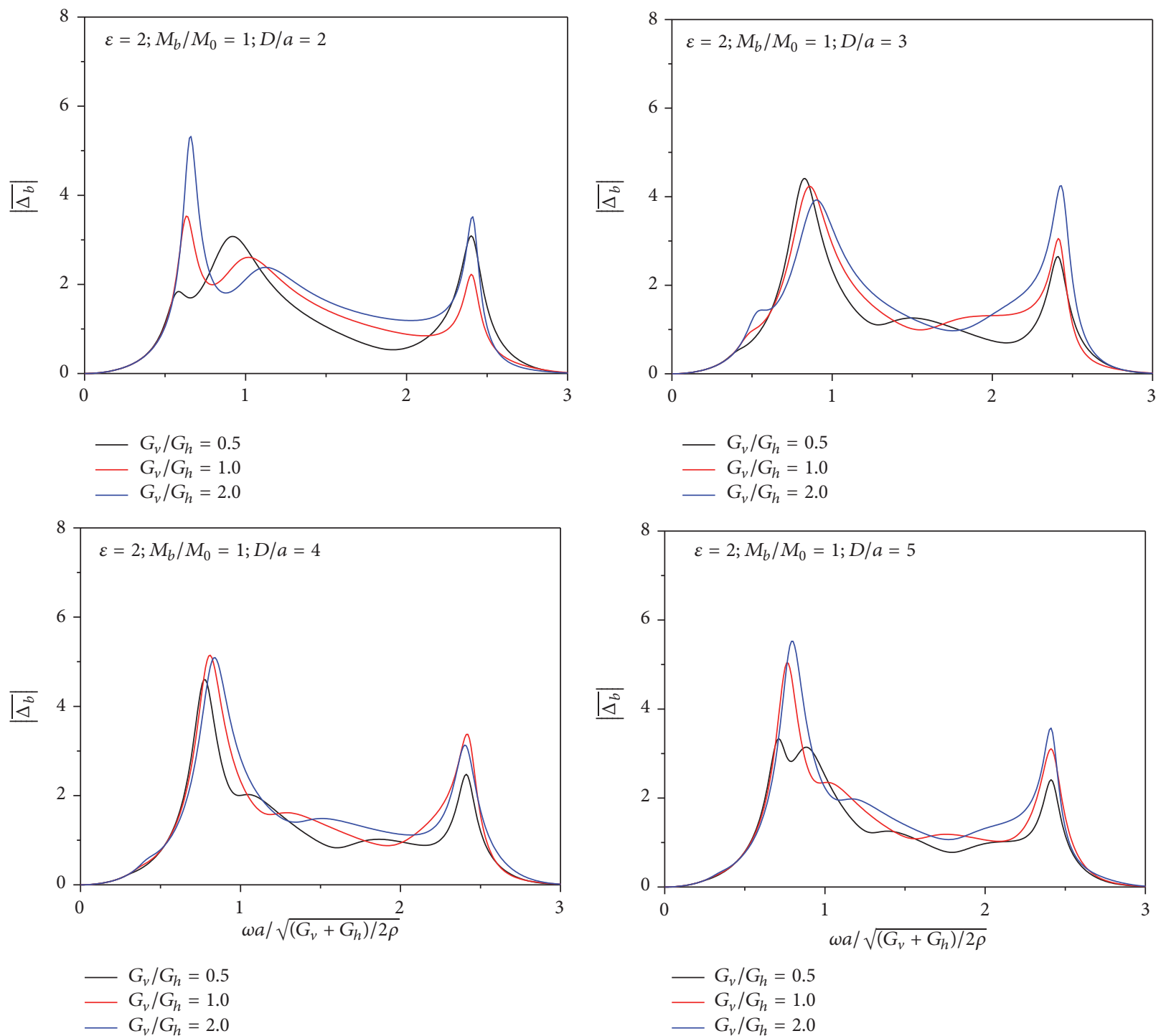

FigURE 7: The superstructure response for different thicknesses of soil layer.

an example, the foundation extends infinitely in the $y$-axis direction with the same cross section. The superstructure is simplified to be a piece of shear wall structure with the same width, and it establishes a rigid connection between the rigid foundation and the TI soil (no slip). Figures 8 and 9 show the effective input motion and the superstructure response. Considering the characteristics of natural soil, there are two conditions selected in multiple TI soil layers: normal-sequence and mixed-sequence. For normal-sequence ground soil, the shear modulus ratio of TI soil layers in the vertical (horizontal) direction is $G_{v 1}^{L}\left(G_{h 1}^{L}\right): G_{v 2}^{L}\left(G_{h 2}^{L}\right): G_{v 3}^{L}\left(G_{h 3}^{L}\right): G_{v 4}^{L}\left(G_{h 4}^{L}\right)=$ $1: 2: 3: 4$; for mixed-sequence ground soil, the shear modulus ratio of TI soil layers in the vertical (horizontal) direction is $G_{v 1}^{L}\left(G_{h 1}^{L}\right): G_{v 2}^{L}\left(G_{h 2}^{L}\right): G_{v 3}^{L}\left(G_{h 3}^{L}\right): G_{v 4}^{L}\left(G_{h 4}^{L}\right)=2: 1: 3: 4$ and $1: 3: 2: 4$. The medium density of soil layers is the same, and the shear modulus ratio of soil layers in a normalsequence and mixed-sequence ground soil in the vertical and horizontal directions is $G_{v i}^{L} / G_{h i}^{L}=0.5(i=1 \sim$
4). The shear modulus of equivalent single TI soil layer $\left(G_{v 1}^{L}\left(G_{h 1}^{L}\right): G_{v 2}^{L}\left(G_{h 2}^{L}\right): G_{v 3}^{L}\left(G_{h 3}^{L}\right): G_{v 4}^{L}\left(G_{h 4}^{L}\right)=1: 1: 1: 1\right)$ in the vertical and horizontal directions shall be solved according to the equivalent shear wave velocity in the corresponding direction.

The bedrock is isotropic, the mass of shear wall is $M_{b} / M_{0}=1$ with rigidity of $\varepsilon=2$, and the density of the bedrock is the same as that of each soil layer. The damping ratio of each soil layer is taken as $\zeta_{i}^{L}\left(\bar{\zeta}^{L}\right)=0.05(i=1 \sim 4)$, and that of the bedrock is $\zeta^{R}=0.02$. The dimensionless frequency is defined as $\omega a / \sqrt{\left(\bar{G}_{v}^{L}+\bar{G}_{h}^{L}\right) / 2 \rho^{L}}$.

4.2.1. The Effective Input Motion. From Figure 8, we can see that there is a difference between the effective input motion in multiple TI soil layers and that in equivalent single TI soil layer. As the soil layer count increases, the superstructure response will exhibit more wave phenomenon. Furthermore, 
TABLE 1: Numerical results of effective input motion for $D / a=2$ and $M_{b} / M_{0}=1$.

\begin{tabular}{cccc}
\hline$\varepsilon$ & $G_{v} / G_{h}$ & First frequency & Peak value \\
\hline \multirow{3}{*}{0} & 0.5 & 0.541 & 1.159 \\
& 1.0 & 0.636 & 1.219 \\
& 2.0 & 0.711 & 1.286 \\
\hline \multirow{3}{*}{2} & 0.5 & 0.536 & 1.410 \\
& 1.0 & 0.606 & 1.697 \\
& 2.0 & 0.636 & 1.988 \\
\hline \multirow{3}{*}{4} & 0.5 & 0.370 & 3.081 \\
& 1.0 & 0.375 & 3.425 \\
& 2.0 & 0.380 & 3.477 \\
\hline
\end{tabular}

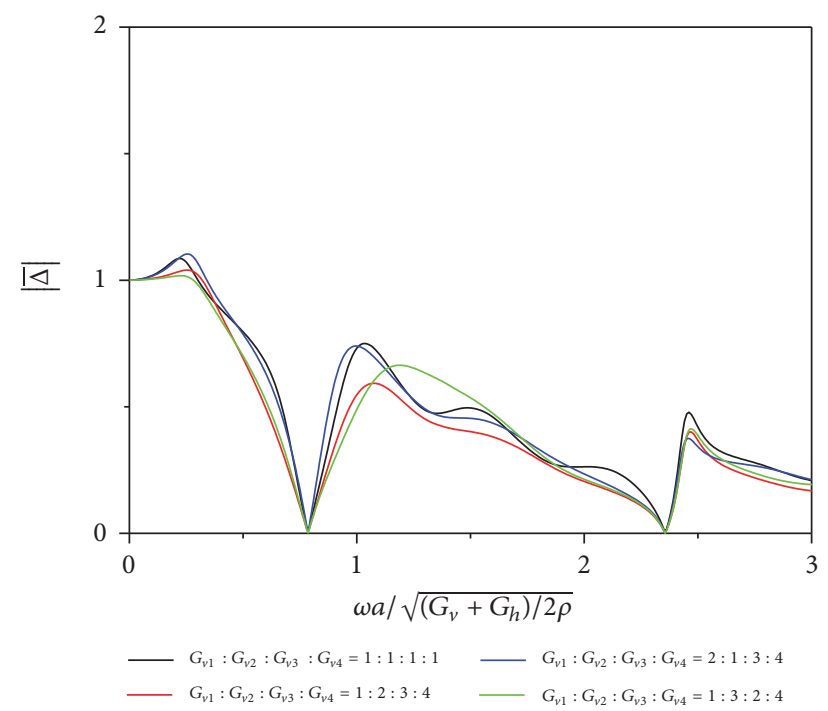

FIGURE 8: The effective input motion in multiple TI soil layers $(\varepsilon=2$, $\left.M_{b} / M_{0}=1\right)$.

the sequence of soil layers has a significant impact on the effective input motion. The effective input motion in mixedsequence layered soil is obviously larger than that in normalsequence layered soil. This illustrates that the effective input motion increases because of a softer layer.

4.2.2. Superstructure Response. It can be seen from Figure 9 that there is a distinction between the superstructure response in multiple TI soil layers and that in equivalent single TI soil layer. In addition, the soil layer sequence has a significant impact on the superstructure response. This indicates that when calculating the superstructure response, the sequence of the actual soil layer shall be considered and shall not be solved simply based on normal-sequence ground soil and single TI soil layer. In general, the peak value of the superstructure response in mixed-sequence TI-layered soil, in single TI soil layer, and in normal-sequence TI-layered soil is successively reduced, while the frequency at the peak value point increases.

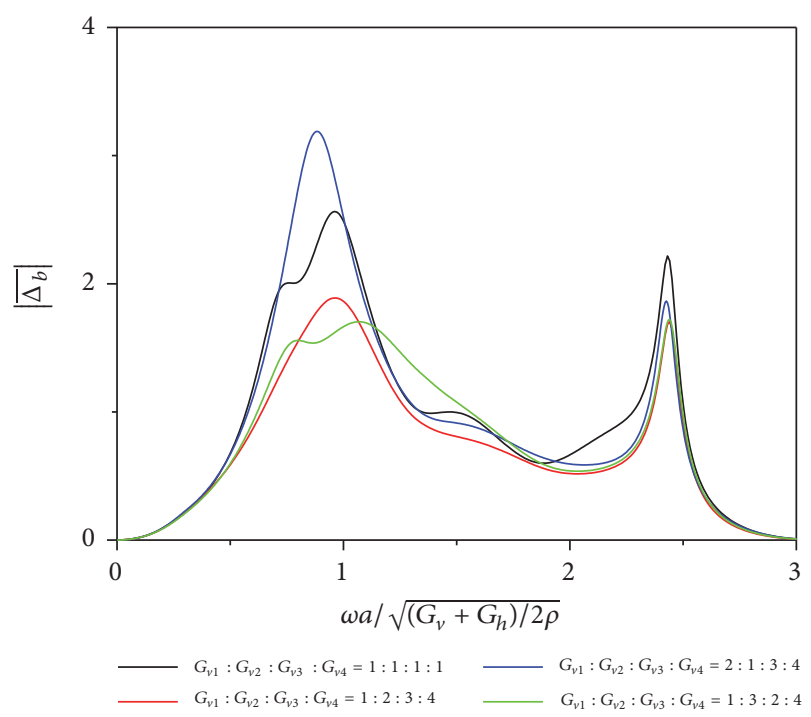

FIGURE 9: The superstructure response in multiple TI soil layers $(\varepsilon=$ $\left.2, M_{b} / M_{0}=1\right)$.

\section{Conclusions}

In this study, the IBEM method is applied to study the effective input motion and the response of the shear wall under the incidence of $\mathrm{SH}$ waves transmitting into layered TI soil. The accuracy of this method is verified by comparing the obtained results with existing research. The semicylindrical foundation-shear wall structure system in single TI soil layer and multiple TI soil layers is utilized as experimental examples. The impact of TI characteristics of soil layers and a shear wall structure on the effective input motion and the superstructure response is studied. The following conclusions are made:

(1) The study on the effective input motion and the superstructure response in single TI soil layer shows that the TI characteristics of the ground soil have a significant impact on the effective input motion and the superstructure response. As the shear modulus ratio of the TI soil layer in the vertical and horizontal directions increases, the peak value of the effective input motion and the superstructure response also increases to a certain degree, and their corresponding peak frequency increases. The increasing flexibility of the upper shear wall structure also increases the effective input motion and the peak value of the superstructure response. In addition, as the mass of superstructure increases, the effective input motion increases while the superstructure response significantly decreases. It is clear that a change in the mass of the superstructure will lead to a change in the seismic energy distribution between the foundation and the superstructure.

(2) The study on the effective input motion and the superstructure response in multiple TI soil layers shows that there is a significant difference between the effective input motion and the superstructure 
response in multiple TI soil layers and that in single TI soil layer. The difference is related to the sequence of the soil layers, and mixed TI soil layers significantly increase the effective input motion and the superstructure response.

\section{Conflicts of Interest}

The authors declare that there are no conflicts of interest regarding the publication of this paper.

\section{Acknowledgments}

This study is supported by the National Natural Science Foundation of China under Grant nos. 51578373 and 51578372 and by the Natural Science Foundation of Tianjin Municipality under Grant no. 16JCYBJC21600, which is gratefully acknowledged.

\section{References}

[1] J. E. Luco, "Dynamic interaction of a shear wall with the soil," Journal of the Engineering Mechanics Division, vol. 95, no. 2, pp. 333-346, 1969.

[2] M. D. Trifunac, "Interaction of a shear wall with the soil for incident plane SH waves," Bulletin of the Seismological Society of America, vol. 62, no. 1, pp. 63-83, 1972.

[3] H. L. Wong and M. D. Trifunac, "Interaction of a shear wall with the soil for incident plane SH waves: elliptical rigid foundation," Bulletin of the Seismological Society of America, vol. 64, no. 6, pp. $1825-1842,1974$.

[4] J. E. Luco, "Torsional response of structures for SH waves: the case of hemispherical foundations," Bulletin of the Seismological Society of America, vol. 66, no. 1, pp. 109-123, 1976.

[5] R. J. Apsel and J. E. Luco, "Torsional response of rigid embedded foundation," Journal of the Engineering Mechanics Division, vol. 102, no. 6, pp. 957-970, 1976.

[6] V. W. Lee, "Investigation of three dimensional soil-structure interaction," Report CE 79-11, Department of Civil Engineering, University of Southern California, Los Angeles, Calif, USA, 1979.

[7] M. I. Todorovska, "In-plane foundation-soil interaction for embedded circular foundations," Soil Dynamics and Earthquake Engineering, vol. 12, no. 5, pp. 283-297, 1993.

[8] M. I. Todorovska and Y. Al Rjoub, "Plain strain soil-structure interaction model for a building supported by a circular foundation embedded in a poroelastic half-space," Soil Dynamics and Earthquake Engineering, vol. 26, no. 6-7, pp. 694-707, 2006.

[9] M. I. Todorovska and Y. A. Rjoub, "Effects of rainfall on soilstructure systemfrequency: examples based on poroelasticity and a comparison with full-scale measurements," Soil Dynamics \& Earthquake Engineering, vol. 26, no. 6-7, pp. 708-717, 2006.

[10] J. Lysmer, "Finite dynamic model for infinite media," Journal of the Engineering Mechanics Division, vol. 95, no. 4, pp. 859-878, 1969.

[11] J. L. Tassoulas and E. Kausel, "On the effect of the rigid sidewall on the dynamic stiffness of embedded circular footings," Earthquake Engineering \& Structural Dynamics, vol. 11, no. 3, pp. 403-414, 1983.
[12] G. Waas, "Earthquake vibration effects and abatement for military facilities," Tech. Rep. S-71-14, Vicksburg, Miss, USA, 1972.

[13] Z. F. Liao, K. Huang, B. Yang et al., "A transmitting boundary for transient wave analyses," Science in China Series A-Mathematics, Physics, Astronomy \& Technological Science, vol. 27, no. 10, pp. 1063-1076, 1969.

[14] W. D. Smith, "A nonreflecting plane boundary for wave propagation problems," Journal of Computational Physics, vol. 15, no. 4, pp. 492-503, 1974.

[15] R. Clayton and B. Engquist, "Absorbing boundary conditions for acoustic and elastic wave equations," Bulletin of the Seismological Society of America, vol. 67, no. 6, pp. 1529-1540, 1977.

[16] B. Engquist and A. Majda, "Absorbing boundary conditions for the numerical simulation of waves," Mathematics of Computation, vol. 31, no. 139, pp. 629-651, 1977.

[17] R. L. Higdon, "Absorbing boundary conditions for difference approximations to the multidimensional wave equation," Mathematics of Computation, vol. 47, no. 176, pp. 437-459, 1986.

[18] J. P. Wolf and G. R. Darbre, "Dynamic-stiffness matrix of soil by the boundary-element method: embedded foundation," Earthquake Engineering \& Structural Dynamics, vol. 12, no. 3, pp. 385-400, 1984.

[19] D. L. Karabalis and D. E. Beskos, "Dynamic response of 3-D rigid surface foundations by time domain boundary element method," Earthquake Engineering \& Structural Dynamics, vol. 12, no. 1, pp. 73-93, 1984.

[20] D. L. Karabalis and D. E. Beskos, "Dynamic response of 3D embedded foundations by the boundary element method," Computer Methods in Applied Mechanics and Engineering, vol. 56, no. 1, pp. 91-119, 1986.

[21] F. C. P. de Barros and J. E. Luco, "Dynamic response of a two-dimensional semi-circular foundation embedded in a layered viscoelastic half-space," Soil Dynamics and Earthquake Engineering, vol. 14, no. 1, pp. 45-57, 1995.

[22] J. Fu and J. Liang, "Influence of site dynamic characteristics on soil-foundation interaction," Earthquake Engineering Engineering Dynamics, 2016.

[23] J. Liang, J. Fu, M. I. Todorovska, and M. D. Trifunac, "Effects of the site dynamic characteristics on soil-structure interaction (I): incident SH-waves," Soil Dynamics and Earthquake Engineering, vol. 44, pp. 27-37, 2013.

[24] J. Liang, J. Fu, M. I. Todorovska, and M. D. Trifunac, "In-plane soil-structure interaction in layered, fluid-saturated, poroelastic half-space I: structural response," Soil Dynamics and Earthquake Engineering, vol. 81, pp. 84-111, 2016.

[25] J. Liang, J. Fu, M. I. Todorovska, and M. D. Trifunac, "In-plane soil-structure interaction in layered, fluid-saturated, poroelastic half-space II: pore pressure and volumetric strain," Soil Dynamics and Earthquake Engineering, vol. 92, pp. 585-595, 2017.

[26] X. Gong, "A preliminary research on anisotropy of the soft clay ground," Journal of Zhejiang University, 1986.

[27] Y. Wang and R. Rajapakse, "Dynamics of rigid strip foundations embedded in orthotropic elastic soils," Earthquake Engineering \& Structural Dynamics, vol. 20, no. 10, pp. 927-947, 1991.

[28] R. K. N. D. Rajapakse and Y. Wang, "Elastodynamic green's functions of orthotropic half plane," Journal of Engineering Mechanics, vol. 117, no. 3, pp. 588-604, 1991.

[29] R. K. N. D. Rajapakse and Y. Wang, "Green's functions for transversely isotropic elastic half space," Journal of Engineering Mechanics, vol. 119, no. 9, pp. 1724-1746, 1993. 
[30] D. J. Kirkner, "Vibration of a rigid disc on a transversely isotropic elastic half space," International Journal for Numerical and Analytical Methods in Geomechanics, vol. 6, no. 3, pp. 293306, 1982.

[31] D.-Z. Wu, Y.-Q. Cai, C.-J. Xu, and H. Zhan, "Torsional vibrations of rigid circular plate on transversely isotropic saturated soil," Applied Mathematics and Mechanics, vol. 27, no. 11, pp. 1541-1548, 2006.

[32] M. Rahimian, M. Eskandari-Ghadi, R. S. Pak, and A. Khojasteh, "Elastodynamic potential method for transversely isotropic solid," Journal of Engineering Mechanics, vol. 133, no. 10, pp. 1134-1145, 2007.

[33] B. A. Ardeshir, G. M. Eskandari, and A. J. Vaseghi, "Analytical solution for a two-layer transversely isotropic half-space affected by an arbitrary shape dynamic surface load," Civil Engineering Infrastructures Journal, vol. 46, no. 1, pp. 1-14, 2013.

[34] Z. Ba, Y. Zhang, and J. Liang, "Amplification of plane SH waves by an alluvial valley based on the TI medium model," Journal of Vibration Engineering, vol. 29, no. 4, 2016.

[35] G. Lin, Z. Han, H. Zhong, and J. Li, "A precise integration approach for dynamic impedance of rigid strip footing on arbitrary anisotropic layered half-space," Soil Dynamics and Earthquake Engineering, vol. 49, pp. 96-108, 2013.

[36] R. Chen, "The response analysis of transversely isotropic elastic strata to incident SH waves," Chinese Quarterly of Mechanics, 1998.

[37] Z. Ba, J. Liang, and Y. Zhang, "Diffraction of SH-waves by topographic features in a layered transversely isotropic halfspace," Earthquake Engineering and Engineering Vibration, vol. 16, no. 1, pp. 11-22, 2017.

[38] G. N. Bycroft, "Soil-foundation interaction and differential ground motions," Earthquake Engineering \& Structural Dynamics, vol. 8, no. 5, pp. 397-404, 1980.

[39] S. M. Day and G. A. Frazier, "Seismic response of hemispherical foundation," Journal of the Engineering Mechanics Division, vol. 105, no. 1, pp. 29-41, 1979.

[40] S.-T. Xue, R. Chen, and L. Qin, "Resonant character of transversely isotropic stratified media," Journal of Tongji University, vol. 30, no. 2, pp. 127-132, 2002. 


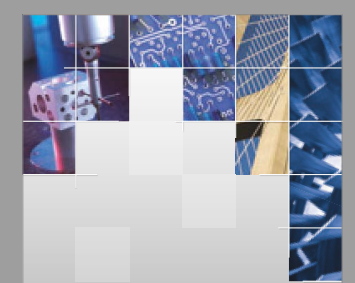

\section{Enfincering}
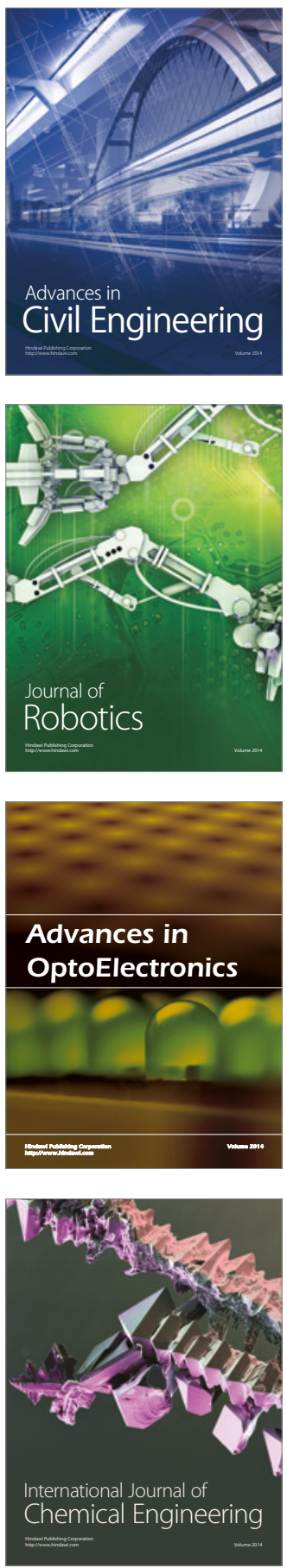

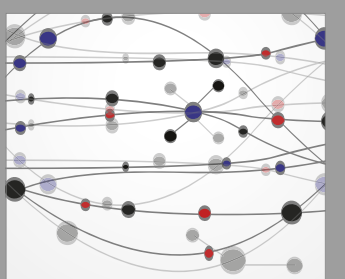

The Scientific World Journal

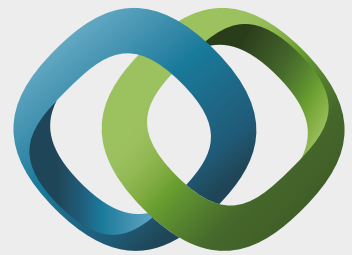

\section{Hindawi}

Submit your manuscripts at

https://www.hindawi.com
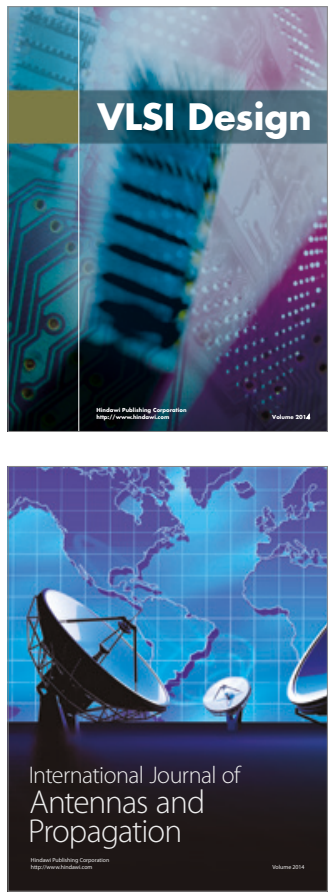

\section{Rotating}

Machinery
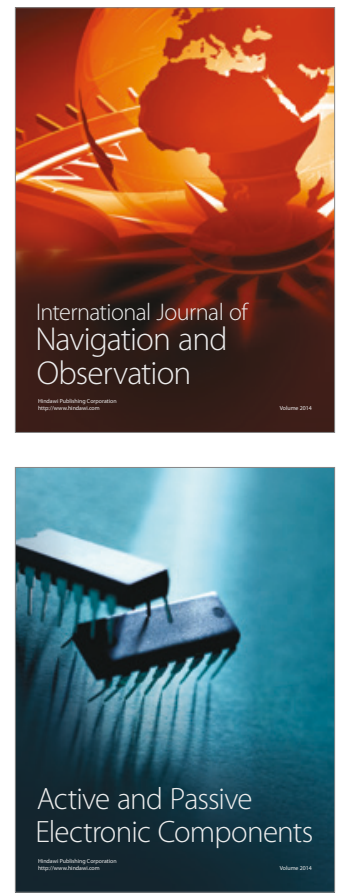
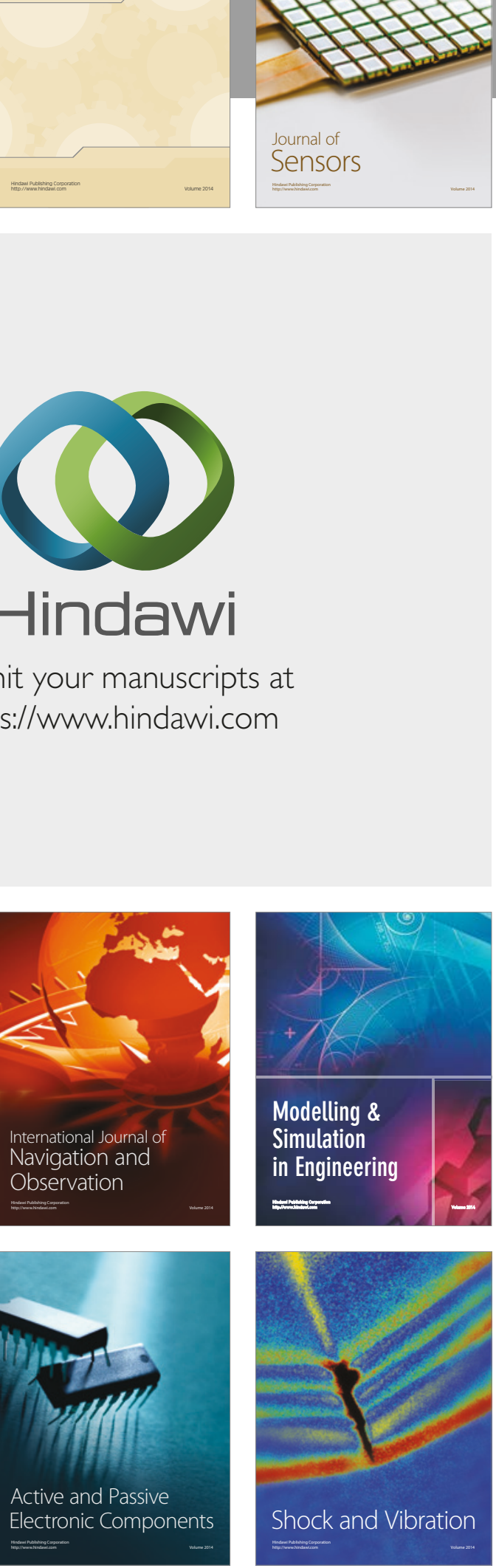
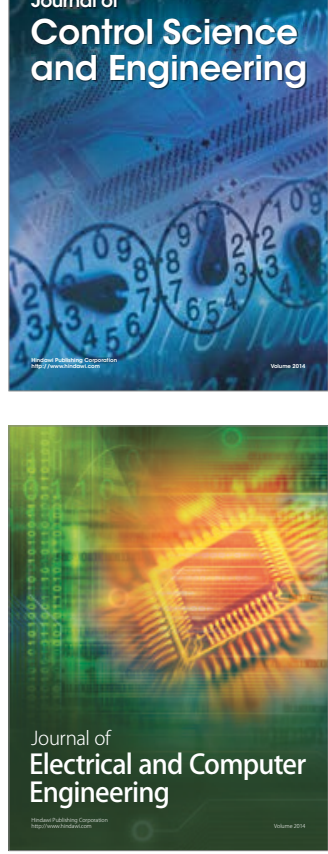

Distributed

Journal of

Control Science

and Engineering
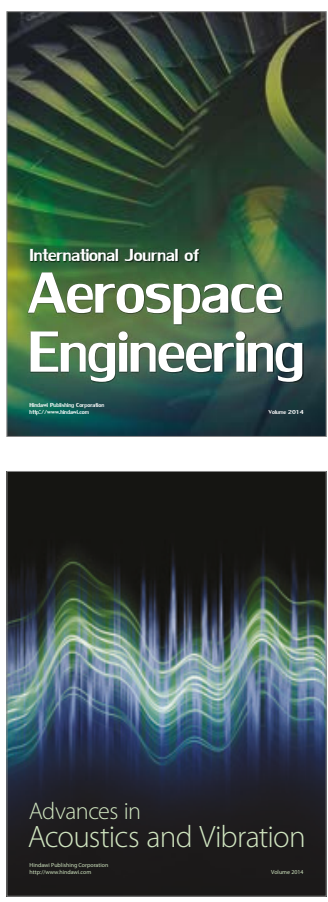

Sensor Networks 